\title{
Photometric catalog of nearby globular clusters ${ }^{\star}$
}

\section{A large homogeneous $(V, I)$ color-magnitude diagram data-base}

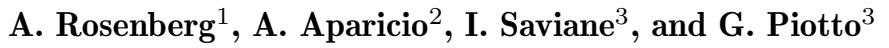 \\ 1 Telescopio Nazionale Galileo, vicolo dell'Osservatorio 5, I-35122 Padova, Italy \\ 2 Instituto de Astrofisica de Canarias, Via Lactea, E-38200 La Laguna, Tenerife, Spain \\ 3 Dipartimento di Astronomia, Univ. di Padova, vicolo dell'Osservatorio 5, I-35122 Padova, Italy
}

Received February 24; accepted June 6, 2000

\begin{abstract}
In this paper we present the second and final part of a large and photometrically homogeneous CCD color-magnitude diagram (CMD) data base, comprising 52 nearby Galactic globular clusters (GGC) imaged in the $V$ and $I$ bands.

The catalog has been collected using only two telescopes (one for each hemisphere). The observed clusters represent $75 \%$ of the known Galactic globulars with $(m-M)_{V} \leq 16.15 \mathrm{mag}$, cover most of the globular cluster metallicity range $(-2.2 \leq[\mathrm{Fe} / \mathrm{H}] \leq-0.4)$, and span Galactocentric distances from $\sim 1.2$ to $\sim 18.5 \mathrm{kpc}$.

In particular, here we present the CMDs for the 13 GGCs observed in the Northern hemisphere. The remaining 39 Southern hemisphere clusters of the catalog have been presented in a companion paper (Rosenberg et al. 2000). We present the first CCD color magnitude diagram for NGC 6779 (M 56).

All the CMDs extend from at least $\sim 2$ magnitudes below the turn-off (i.e. $V_{\lim } \geq 22$ ) to the tip of the red giant branch. The calibration has been done using a large number of standard stars, and the absolute calibration is reliable to $\mathrm{a} \sim 0.02 \mathrm{mag}$ level in both filters.

This catalog, because of its homogeneity, is expected to represent a useful data base for the measurement of the main absolute and relative parameters characterizing the CMD of GGCs.
\end{abstract}

Key words: astronomical data base: miscellaneous catalogs — stars: Hertzsprung-Russell (HR) and C-M diagrams - stars: population II - globular clusters: general

Send offprint requests to: A. Rosenberg, e-mail: alf@ll.iac.es

* Based on observations made with the $1 \mathrm{~m}$ Jacobus Kapteyn Telescope operated on the island of La Palma by the ING in the Spanish Observatorio del Roque de Los Muchachos of the Instituto de Astrofisica de Canarias.

\section{Introduction}

As discussed in Rosenberg et al. (2000, hereafter Paper I), the heterogeneity of the data often used in the literature for large scale studies of the Galactic globular cluster (GGC) properties has induced us to start a large survey of both southern and northern GGCs by means of 1-m class telescopes, i.e. the $91 \mathrm{~cm}$ European Southern Observatory (ESO)/Dutch telescope and the $1 \mathrm{~m}$ Isaac Newton Group (ING)/Jacobus Kapteyn telescope (JKT). We were able to collect the data for 52 of the 69 known GGCs with $(m-M)_{V} \leq 16.15$. Thirty-nine objects have been observed with the Dutch Telescope and the data have been already presented in Paper I. The images and the photometry of the remaining 13 GGCs, observed with the JKT are presented in this paper. A graphical representation of the spatial distribution of our cluster sample is given in Fig. 1.

As a first exploitation of this new data base, we have conducted a GGC relative age investigation based on the best 34 CMDs of our catalog (Rosenberg et al. 1999, hereafter Paper III), showing that most of the GGCs have the same age. We have also used the data base to obtain a photometric metallicity ranking scale (Saviane et al. 2000, hereafter Paper IV), based on the red giant branch (RGB) morphology.

There are many other parameters that can be measured from an homogeneous, well calibrated CMD data base: the horizontal branch (HB) level, homogeneous reddening and distance scales, etc. We are presently working on these problems. However, we believe it is now the time to present to the community the complete data base to give to anyone interested the opportunity to take advantage of it.

In the next section, we will describe the observations collected at the JKT in 1997. The data reduction and calibration is presented in Sect. 3, where a comparison of the calibration of the northern and southern clusters is also 


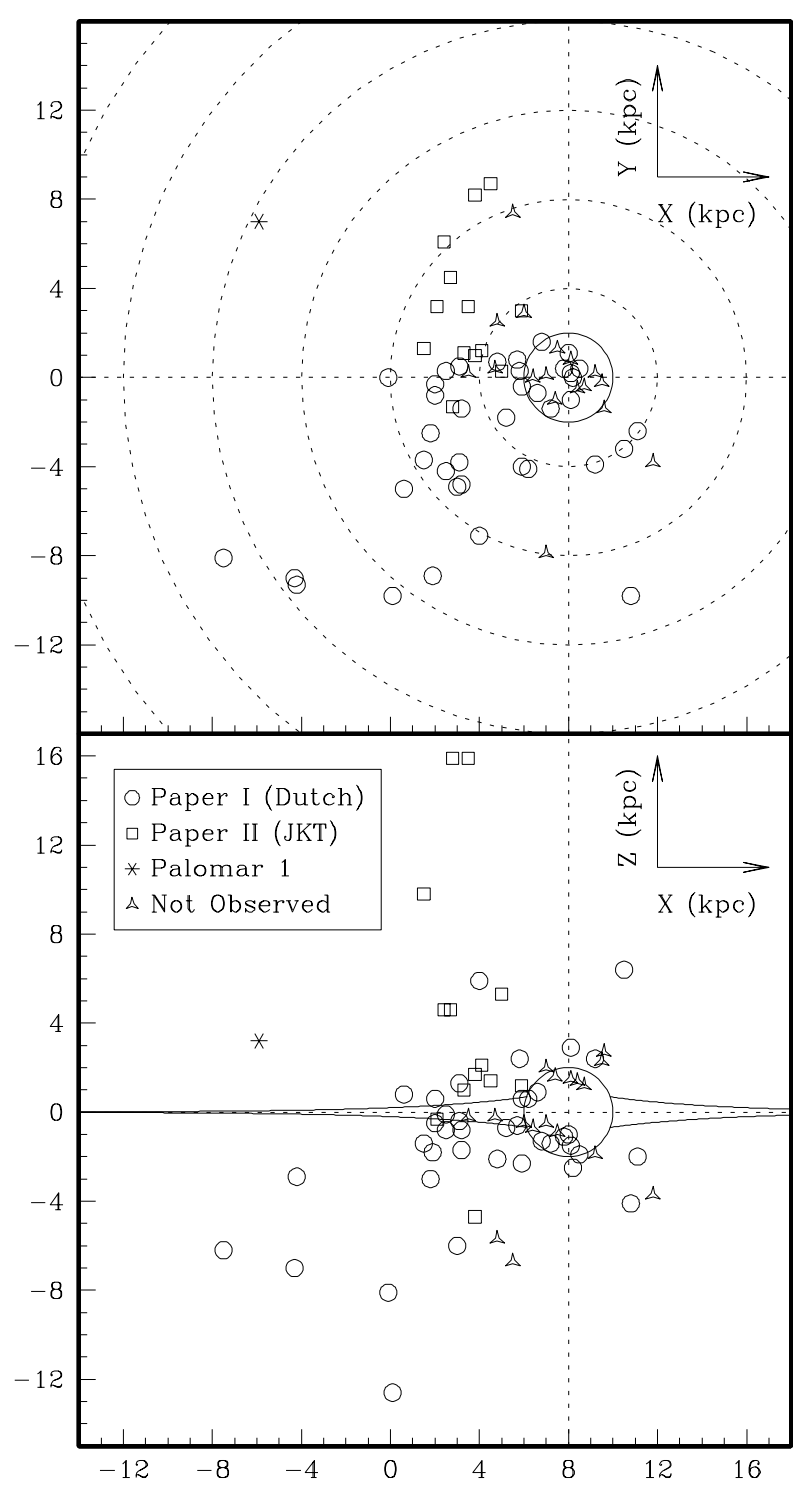

Fig. 1. Galactocentric distribution of all the GGCs with $(m-$ $M)_{V} \leq 16.15 \mathrm{mag}$. In the upper panel, the projection of the GGC location over the Galactic plane is presented. The open circles represent the GGCs presented in Paper I, the open squares the GGCs whose photometry and CMDs are discussed in this paper, and the asterisk indicates the position of the GGC Pal 1 (Rosenberg et al. 1998). The clusters which are not presented in our catalog are marked by open triangles. The lower panel shows the location of the same GGCs in a plane perpendicular to the Galactic plane. The Milky Way is also schematically represented

discussed for three objects observed with both telescopes. In order to assist the reader, in Sect. 4 we present the main parameters characterizing our clusters. Finally, the observed fields for each cluster, and the obtained CMDs are presented and briefly discussed in Sect. 5 .

\section{Observations}

The data were collected on May 30-June 2 1997. For almost 2 nights we had quite good seeing conditions (FWHM between 0.65 and 0.85 arcsec). Unfortunately, too few standards were observed during this run. In order to ensure an homogeneous calibration, we reobserved all the same cluster fields (plus numerous standard fields) in June 24, 1998, a night that was photometric and with a stable seeing.

Observations were done at the $1 \mathrm{~m}$ Jacobus Kapteyn telescope (JKT), located at the Roque de Los Muchachos Observatory. The same instrumentation was used in both runs: A thinned Tektronix CCD with $1024 \times 1024$ pixels projecting 0.331 arcsec on the sky and providing a field of view of 5.6 arcmin square. The detector system was linear to $0.1 \%$ over the full dynamic range of the 16 bit analog-to-digital converter.

Three short (10 s), two medium (45 s $\div 120 \mathrm{~s})$ and one large $(1800 \mathrm{~s})$ exposures were taken for one field of each of the proposed objects in each filter. Also a large number of standard stars were obtained during the second observing run. This was done in order to have all the clusters in exactly the same system, making internal errors negligible.

\section{Data reduction and calibration}

The data were reduced following the same procedure described in Paper I.

The absolute calibration of the observations is based on a set of standard stars of the catalog of Landolt (1992). Eighteen standard stars were observed; specifically, the observed fields were PG1525, PG1633, Mark-A and PG2213. At least 4 exposures at different airmasses were taken during the night for each standard field, making a total of $\sim 80$ individual measures per filter.

Thanks to the relatively stable seeing conditions, for the aperture photometry we used for all the standards (and the cluster fields) a 12 pixel aperture ( 4 arcsec). The aperture magnitudes were normalized by correcting for the exposure time and airmass. Using the standards observed at different airmasses, we estimated for the extinction coefficients in the two filters: $A_{V}=0.11$ and $A_{I}=0.06$. For the calibration curves we adopted a linear relation. The best fitting straight lines are:

$V-v(\operatorname{ap})=(0.017 \pm 0.003) \times(V-I)-(2.232 \pm 0.002)$; $I-i(\mathrm{ap})=(0.053 \pm 0.003) \times(V-I)-(2.679 \pm 0.002)$.

The magnitude differences vs. the standard color are plotted in Fig. 2, where the solid lines represent the above equations.

\subsection{Comparison between the two catalogs}

Apart from the 13 GGCs presented in the following sections, we re-observed three additional clusters selected from the southern hemisphere catalog presented in Paper I. We covered the same spatial region, in order 


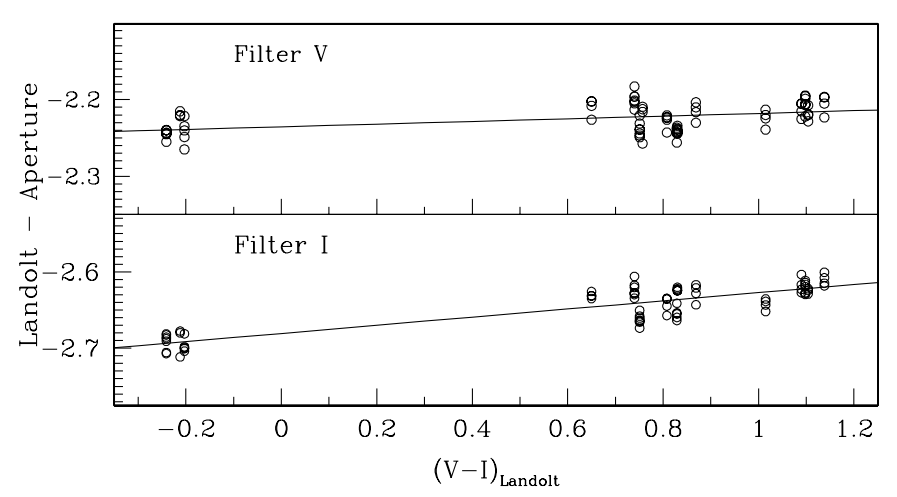

Fig. 2. Calibration curves for the $V$ (upper panel) and $I$ (lower panel) filters for the June $24^{\text {th }} 1998$ run

to test the homogeneity of the two catalogs created using the two different telescopes.

In Fig. 3 the magnitudes of 170 stars (with photometric error smaller than $0.025 \mathrm{mag}$ and $V$ magnitude brighter than 18) in NGC 5897 from the JKT and the ESO/Dutch telescope data sets are compared. The differences in magnitude and color between the two observing runs are: $0.00 \pm 0.02,-0.01 \pm 0.02$ and $0.01 \pm 0.02$ in $V, I$ and $V-I$, respectively. For the other two clusters, we have: $\Delta V_{\mathrm{DUT}}^{\mathrm{JKT}}=0.02 \pm 0.02, \Delta I_{\mathrm{DUT}}^{\mathrm{JKT}}=0.01 \pm 0.02$ and $\Delta(V-I)_{\mathrm{DUT}}^{\mathrm{JKT}}=0.01 \pm 0.02$ for 163 stars selected in $\mathrm{NGC} 6093$, and $\Delta V_{\mathrm{DUT}}^{\mathrm{JKT}}=0.01 \pm 0.02, \Delta I_{\mathrm{DUT}}^{\mathrm{JKT}}=0.00 \pm 0.02$ and $\Delta(V-I)_{\mathrm{DUT}}^{\mathrm{JKT}}=0.01 \pm 0.02$ for 249 stars selected in NGC 6171 (selection criteria as in the case of NGC 5897). Moreover, the slopes of the color differences vs. both the magnitude and the color are always $\leq 0.001 \pm 0.002$. Therefore, the two catalogs must be considered photometrically homogeneous and the measures of the absolute and relative parameters of the CMDs perfectly compatible.

\section{Parameters for the GGC sample}

In order to assist the reader, we present in Tables 1, 2 and 3 the basic parameters available for our GGC sample ${ }^{1}$.

In Table 1 we give the coordinates, the position, and the metallicity of the clusters: right ascension and declination (epoch J2000, Cols. 3 and 4); Galactic longitude and latitude (Cols. 5 and 6); Heliocentric (Col. 7) and Galactocentric (Col. 8) distances (assuming $R_{\odot}=$ $8.0 \mathrm{kpc}$ ); spatial components $(X, Y, Z)$ (Cols. 9, 10 and 11 ) in the Sun-centered coordinate system ( $X$ pointing toward the Galactic center, $Y$ in direction of Galactic rotation, $Z$ toward North Galactic Pole) and, finally, the metallicity given in Rutledge et al. (1997), on both the Zinn \& West (1984) and Carretta \& Gratton (1997) scales.

\footnotetext{
${ }^{1}$ Unless otherwise stated, the data presented in these tables are taken from the McMaster catalog described by Harris (1996).
}
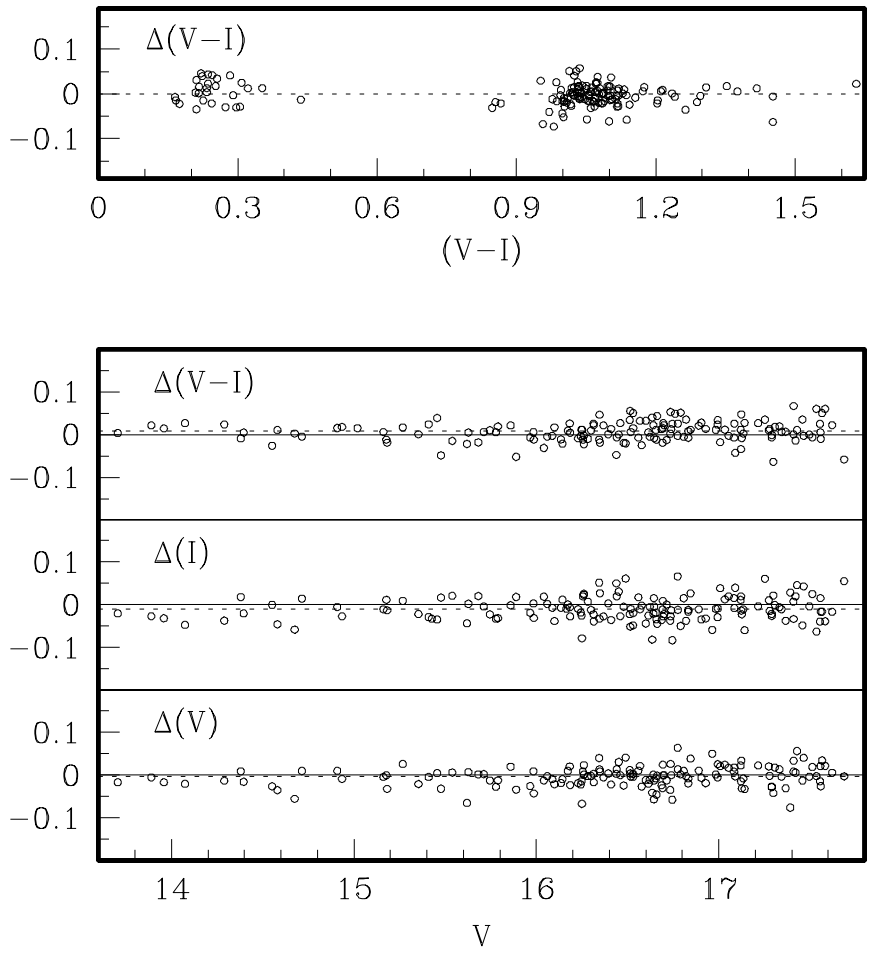

Fig. 3. Comparison between the JKT and the ESO/Dutch telescope magnitudes for the 170 stars in NGC 5897 with errors $\leq 0.025$. The differences in magnitude and color between the two runs are: $0.00 \pm 0.02,-0.01 \pm 0.02$ and $0.01 \pm 0.02$ in $V, I$ and $V-I$, respectively. Notice also that the slopes of the differences in color vs. magnitudes or color of one of the runs (upper panel), are negligible $(\leq 0.001 \pm 0.002)$, making the catalogs photometrically homogeneous

In Table 2, the photometric parameters are given. Col. 3 lists the foreground reddening; Col. 4, the $V$ magnitude level of the horizontal branch; Col. 5, the apparent visual distance modulus; integrated $V$ magnitudes of the clusters are given in Col. 6; Col. 7 gives the absolute visual magnitude. Columns 8 to 11 give the integrated color indices (uncorrected for reddening). Column 12 gives the specific frequency of RR Lyrae variables, while Col. 13 list the horizontal-branch morphological parameter (Lee 1990).

In Table 3, we present the kinematical and structural parameters for the observed clusters. Column 3 gives the heliocentric radial velocity $\left(\mathrm{km} \mathrm{s}^{-1}\right)$ with the observational (internal) uncertainty; Col. 4 , the radial velocity relative to the local standard of rest; Col. 5, the concentration parameter $\left(c=\log \left(r_{\mathrm{t}} / r_{\mathrm{c}}\right)\right)$; a "c" denotes a corecollapsed cluster; Cols. 6 and 7 , the core and the half mass radii in arcmin; Col. 8, the logarithm of the core relaxation time, in years; and Col. 9 the logarithm of the relaxation time at the half mass radius. Column 10, the central surface brightness in $V$; and Col. 11, the logarithm of central luminosity density (Solar luminosities per cubic parsec). 
Table 1. Identifications, positional data and metallicity for the observed clusters

\begin{tabular}{|c|c|c|c|c|c|c|c|c|c|c|c|c|}
\hline \multirow[t]{2}{*}{ ID } & \multirow[t]{2}{*}{ Cluster } & \multirow{2}{*}{$\begin{array}{c}\mathrm{AR}^{\mathrm{a}} \\
\left(\mathrm{h} \mathrm{m} \mathrm{s}^{2}\right)\end{array}$} & $\mathrm{DEC}^{\mathrm{b}}$ & \multirow{2}{*}{$\begin{array}{c}l^{\mathrm{c}} \\
\left(^{\mathrm{O}}\right)\end{array}$} & \multirow{2}{*}{$\begin{array}{l}b^{\mathrm{d}} \\
\left(^{\mathrm{O}}\right)\end{array}$} & \multirow{2}{*}{$\begin{array}{c}R_{\odot}^{\mathrm{e}} \\
(\mathrm{kpc})\end{array}$} & \multirow{2}{*}{$\begin{array}{c}R_{\mathrm{GC}}^{\mathrm{f}} \\
(\mathrm{kpc})\end{array}$} & \multirow{2}{*}{$\begin{array}{c}X^{\mathrm{g}} \\
(\mathrm{kpc})\end{array}$} & \multirow{2}{*}{$\begin{array}{c}Y^{\mathrm{h}} \\
(\mathrm{kpc})\end{array}$} & \multirow{2}{*}{$\begin{array}{c}Z^{\mathrm{i}} \\
(\mathrm{kpc})\end{array}$} & \multicolumn{2}{|c|}{$[\mathrm{Fe} / \mathrm{H}]$} \\
\hline & & & $\left(\begin{array}{lll}0 & 1 & \prime \prime\end{array}\right)$ & & & & & & & & $\mathrm{ZW} 84^{\mathrm{j}}$ & CG97 ${ }^{\mathrm{k}}$ \\
\hline 1 & NGC 5053 & 131627.0 & +174153 & 335.69 & +78.94 & 16.2 & 16.8 & +2.8 & -1.3 & +15.9 & -2.10 & -1.78 \\
\hline 2 & NGC 5272 & 134211.2 & +282232 & 042.21 & +78.71 & 10.0 & 11.9 & +1.5 & +1.3 & +9.8 & $-1.66^{1}$ & $-1.34^{2}$ \\
\hline 3 & NGC 5466 & 140527.3 & +283204 & 042.15 & +73.59 & 16.6 & 16.9 & +3.5 & +3.2 & +15.9 & $-2.22^{1}$ & - \\
\hline 4 & NGC 5904 & 151833.8 & +020458 & 003.86 & +46.80 & 7.3 & 6.1 & +5.0 & +0.3 & +5.3 & -1.38 & -1.12 \\
\hline 5 & NGC 6205 & 164141.5 & +362737 & 059.01 & +40.91 & 7.1 & 8 . & +2.7 & +4.5 & +4.6 & -1.63 & -1.33 \\
\hline 6 & NGC 6218 & $1647 \quad 14.5$ & -015652 & 015.72 & +26.31 & 4.7 & 4.6 & +4.1 & +1.2 & +2.1 & -1.40 & -1.14 \\
\hline 7 & NGC 6254 & 165708.9 & -040558 & 015.14 & +23.08 & 4.3 & 4.6 & +3.8 & +1.0 & +1.7 & -1.55 & -1.25 \\
\hline 8 & NGC 6341 & $17 \quad 1707.3$ & +430811 & 068.34 & +34.86 & 8.1 & 9.5 & +2.4 & +6.1 & +4.6 & $-2.24^{1}$ & $-2.16^{2}$ \\
\hline 9 & NGC 6366 & 172744.3 & -050436 & 018.41 & +16.04 & 3.6 & 4.9 & +3.3 & +1.1 & +1.0 & -0.58 & -0.73 \\
\hline 10 & NGC 6535 & 180350.7 & -00 1749 & 027.18 & +10.44 & 6.8 & 3.9 & +5.9 & +3.0 & +1.2 & -1.78 & -1.51 \\
\hline 11 & NGC 6779 & 191635.5 & +301105 & 062.66 & +08.34 & 9.9 & 9.5 & +4.5 & +8.7 & +1.4 & $-1.94^{1}$ & - \\
\hline 12 & NGC 6838 & 195346.1 & +184642 & 056.74 & -04.56 & 3.8 & 6.7 & +2.1 & +3.2 & -0.3 & -0.58 & -0.73 \\
\hline 13 & NGC 7078 & 212958.3 & +121001 & 065.01 & -27.31 & 10.2 & 10.3 & +3.8 & +8.2 & -4.7 & -2.13 & -2.02 \\
\hline
\end{tabular}

In the following cases, the $[\mathrm{Fe} / \mathrm{H}]$ values were taken directly from $\left({ }^{1}\right)$ ZW84 and $\left(^{2}\right)$ CG97.

${ }^{a}$ Right Ascension (2000)

b Declination (2000)

c Galactic Longitude

${ }^{\mathrm{d}}$ Galactic Latitude

e Heliocentric Distance

${ }^{f}$ Galactocentric Distance
Sun-Centered coordinates: ${ }^{g} X$ : Toward the Galactic Center

h $Y$ : in direction of Galactic rotation

${ }^{\text {i }} Z$ : Towards North Galactic Plane

[Fe/H] (From Rutledge et al. 1997): ${ }^{j}$ in the ZW84 scale

k in the CG97 scale.

Table 2. Photometric parameters

\begin{tabular}{rccccccccccrr}
\hline ID & Cluster & $E(B-V)^{\mathrm{a}}$ & $V_{\mathrm{HB}}^{\mathrm{b}}$ & $(m-M)_{V}^{\mathrm{c}}$ & $V_{\mathrm{t}}^{\mathrm{d}}$ & $M v_{\mathrm{t}}^{\mathrm{e}}$ & $U-B^{\mathrm{f}}$ & $B-V^{\mathrm{f}}$ & $V-R^{\mathrm{f}}$ & $V-I^{\mathrm{f}}$ & $S_{\mathrm{RR}}^{\mathrm{g}}$ & $H B R^{\mathrm{h}}$ \\
\hline 1 & NGC 5053 & 0.03 & 16.70 & 16.14 & 9.47 & -6.67 & 0.03 & 0.65 & - & 0.90 & 21.4 & 0.52 \\
2 & NGC 5272 & 0.01 & 15.58 & 15.04 & 6.19 & -8.85 & 0.09 & 0.69 & - & 0.93 & 75.3 & 0.08 \\
3 & NGC 5466 & 0.00 & 16.60 & 16.10 & 9.04 & -7.06 & 0.00 & 0.67 & - & 0.82 & 41.9 & 0.58 \\
4 & NGC 5904 & 0.03 & 15.00 & 14.41 & 5.65 & -8.76 & 0.17 & 0.72 & 0.45 & 0.95 & 38.4 & 0.31 \\
\hline 5 & NGC 6205 & 0.02 & 14.95 & 14.28 & 5.78 & -8.50 & 0.02 & 0.68 & - & 0.86 & 2.0 & 0.97 \\
6 & NGC 6218 & 0.19 & 14.70 & 13.97 & 6.70 & -7.27 & 0.20 & 0.83 & 0.56 & 1.14 & 0.0 & 0.97 \\
7 & NGC 6254 & 0.28 & 15.05 & 14.03 & 6.60 & -7.43 & 0.23 & 0.90 & 0.53 & 1.21 & 1.1 & 0.98 \\
8 & NGC 6341 & 0.02 & 15.20 & 14.59 & 6.44 & -8.15 & 0.01 & 0.63 & - & 0.88 & 13.7 & 0.91 \\
\hline 9 & NGC 6366 & 0.69 & 15.65 & 14.92 & 9.20 & -5.72 & 0.97 & 1.44 & - & - & 5.1 & -0.97 \\
10 & NGC 6535 & 0.32 & 15.80 & 15.15 & 10.47 & -4.68 & 0.29 & 0.94 & - & - & 0.0 & 1.00 \\
11 & NGC 6779 & 0.20 & 16.30 & 15.60 & 8.27 & -7.33 & 0.15 & 0.86 & - & 1.16 & 2.3 & 0.98 \\
12 & NGC 6838 & 0.25 & 14.50 & 13.70 & 8.19 & -5.51 & 0.63 & 1.09 & 0.63 & 1.36 & 0.0 & -1.00 \\
\hline 13 & NGC 7078 & 0.09 & 15.90 & 15.31 & 6.20 & -9.11 & 0.06 & 0.68 & - & 0.85 & 25.4 & 0.67 \\
\hline
\end{tabular}

${ }^{\text {a }}$ Foreground reddening

${ }^{\mathrm{b}}$ HB Level (our measures)
e Absolute visual magnitude
${ }^{\mathrm{f}}$ Integrated color indices
g Specific frequency of RR Lyrae variables
${ }^{\mathrm{h}} \mathrm{HB}$ ratio: $H B R=(B-R) /(B+V+R)$.

c Apparent visual distance modulus

\section{The Color-Magnitude Diagrams}

In this section the $V$ vs. $(V-I)$ CMDs for the 13 GGCs are presented.

The same color and magnitude scales have been used in plotting the CMDs, so that differential measurements can be done directly using the plots. The adopted scale is the same used in previous Paper I. Two dot sizes have been used: the larger ones correspond to the better measured stars, normally selected on the basis of their photometric error $(\leq 0.1)$ and sharpness parameter. In some exceptional cases, a selection on the radial distance from the cluster center is also done, in order to make more evident the cluster CMD over the field stars, or to show differential reddening effects. The smaller size dots show all the measured stars with errors (as calculated by DAOPHOT) smaller than 0.15 mag. 
Table 3. Kinematics, and structural parameters

\begin{tabular}{rlrrrrrrrrc}
\hline ID & Cluster & $V_{\mathrm{r}}^{\mathrm{a}}$ & $V_{\mathrm{LSR}}^{\mathrm{b}}$ & $c^{\mathrm{c}}$ & $r_{\mathrm{c}}^{\mathrm{d}}$ & $r_{\mathrm{h}}^{\mathrm{e}}$ & $\lg \left(t_{\mathrm{c}}\right)^{\mathrm{f}}$ & $\lg \left(t_{\mathrm{h}}\right)^{\mathrm{g}}$ & $\mu_{V}^{\mathrm{h}}$ & $\rho_{0}^{\mathrm{i}}$ \\
\hline 1 & NGC 5053 & $+44.0 \pm 0.4$ & +51.8 & 0.82 & 2.25 & 3.50 & 9.87 & 9.59 & 22.19 & 0.47 \\
2 & NGC 5272 & $-148.6 \pm 0.4$ & -138.0 & 1.85 & 0.50 & 1.12 & 8.78 & 9.02 & 16.34 & 3.56 \\
3 & NGC 5466 & $+107.7 \pm 0.3$ & 119.7 & 1.43 & 1.96 & 2.25 & 9.75 & 9.37 & 21.28 & 0.78 \\
4 & NGC 5904 & $+52.1 \pm 0.5$ & +65.2 & 1.87 & 0.40 & 2.11 & 8.30 & 9.13 & 16.05 & 3.94 \\
\hline 5 & NGC 6205 & $-246.6 \pm 0.9$ & -228.2 & 1.49 & 0.88 & 1.49 & 8.94 & 8.96 & 16.80 & 3.32 \\
6 & NGC 6218 & $-42.1 \pm 0.6$ & -26.3 & 1.38 & 0.66 & 2.16 & 8.23 & 8.86 & 18.17 & 3.27 \\
7 & NGC 6254 & $+75.8 \pm 1.0$ & +91.5 & 1.40 & 0.86 & 1.81 & 8.38 & 8.56 & 17.69 & 3.51 \\
8 & NGC 6341 & $-121.6 \pm 1.5$ & -103.0 & 1.81 & 0.23 & 1.09 & 7.84 & 8.56 & 15.58 & 4.30 \\
\hline 9 & NGC 6366 & $-122.3 \pm 0.5$ & -106.3 & 0.92 & 1.83 & 2.63 & 8.77 & 8.50 & 21.24 & 2.39 \\
10 & NGC 6535 & $-215.1 \pm 0.5$ & -198.0 & 1.30 & 0.42 & 0.77 & 7.47 & 8.05 & 20.22 & 2.66 \\
11 & NGC 6779 & $-135.7 \pm 0.8$ & -116.9 & 1.37 & 0.37 & 1.16 & 8.27 & 8.70 & 18.06 & 3.27 \\
12 & NGC 6838 & $-22.9 \pm 0.2$ & -5.5 & 1.15 & 0.63 & 1.65 & 7.62 & 8.10 & 19.22 & 3.06 \\
\hline 13 & NGC 7078 & $-107.5 \pm 0.3$ & -95.0 & 2.50 & 0.07 & 1.06 & 7.10 & 9.01 & 14.21 & 5.37 \\
\hline
\end{tabular}

The images of the fields are oriented with North at the top and East on the left side. Each field covers 5!6 square. The same spatial scale has been used in all the cluster images.

In the next subsections, we briefly present the single CMDs and clusters, and give some references to the best existing CMDs. This does not pretend to be a complete bibliographical catalog: a large number of CMDs are available in the literature for many of the clusters of this survey; we will concentrate just on the best CCD photometric works. The tables with the position and photometry of the measured stars will be available on-line at the IAC (http://www.iac.es/proyect/poblestelares) and Padova (http://menhir.pd.astro.it/).

NGC 5053. (Fig. 4)

NGC 5053 is a low concentration cluster, and, as all the sparse clusters, it has a small central velocity dispersion and central mass density. It is one of the clusters farthest from the Galactic center in our sample. NGC 5053 is one of the most metal-poor clusters in our Galaxy (Sarajedini \& Milone 1995).

There are several CMD studies for NGC 5053 in the literature. Nemec \& Cohen (1989) presented the first CCD CMD in the Thuan \& Gunn (1976) $g$ and $r$ filters, reaching $g \simeq 23$. Their CMD is one magnitude deeper than ours, but the stellar distribution and number of stars above the $22^{\text {nd }}$ magnitude is almost the same in both diagrams. The upper part of their RGB (above the HB) is saturated. Heasley \& Christian (1991a) present $B$ and $V$ photometry extended to $V \simeq 22$. Their CMD is poorly populated, presenting only a few stars in the $\mathrm{HB}$ region. Their upper RGB is also truncated at $V \sim 15.5$. In the same year, Fahlman et al. (1991) present a study of the stellar content and structure of this cluster, including a $B$ and $V$ CMD that reaches $V=24$. They are mainly interested in the stellar content and structure, being most of the data obtained just for the $V$ band, and only one field (field \#2) in both, $V$ and $B$ bands. The corresponding CMD is deep but poorly populated, presenting $\sim 6$ stars in their HB.

More recently, Sarajedini and Milone (1995) present $B, V$, and $I$ photometry for the upper part of the CMD (above the cluster's TO), making a good sampling (25\% larger than ours) of the evolved cluster stars.

We present a photometry $(\sim 5300$ stars, seeing of $\sim 1.1^{\prime \prime}$ ) that covers the cluster from the brighter RGB stars down to the $22^{\text {nd }}$ magnitude. All the CMD sequences are well defined, including a blue straggler sequence. NGC 5053 has a BHB with a few RR-Lyrae and also a few stars in the red side of the HB (evolved HB stars?).

\section{NGC 5272 (M 3). (Fig. 5)}

The northern "standard couple" of clusters affected by the second parameter effect is represented by M 3 and M 13 . M 3 has a well populated HB, both on the red and blue sides, while the M $13 \mathrm{HB}$ is populated only on the blue side. This is a typical example where the age cannot be advocated as the (unique) responsible of the differences in the HB morphology (Paper III, Johnson \& Bolte 1998; Davidge \& Courteau 1999).

Very recently, M 3 has been the subject of many studies. Laget et al. (1998) present $U V, U, V$ and $I$ photometry of the central part of M 3 from HST data. Their CMDs reach $V \sim 20$, and is extremely well defined. Their stellar sample is smaller than our one, but clearly shows the mean regions of the CMD. Its overall structure is very similar to our "selected" diagram. Kaluzny et al. (1998) look for 

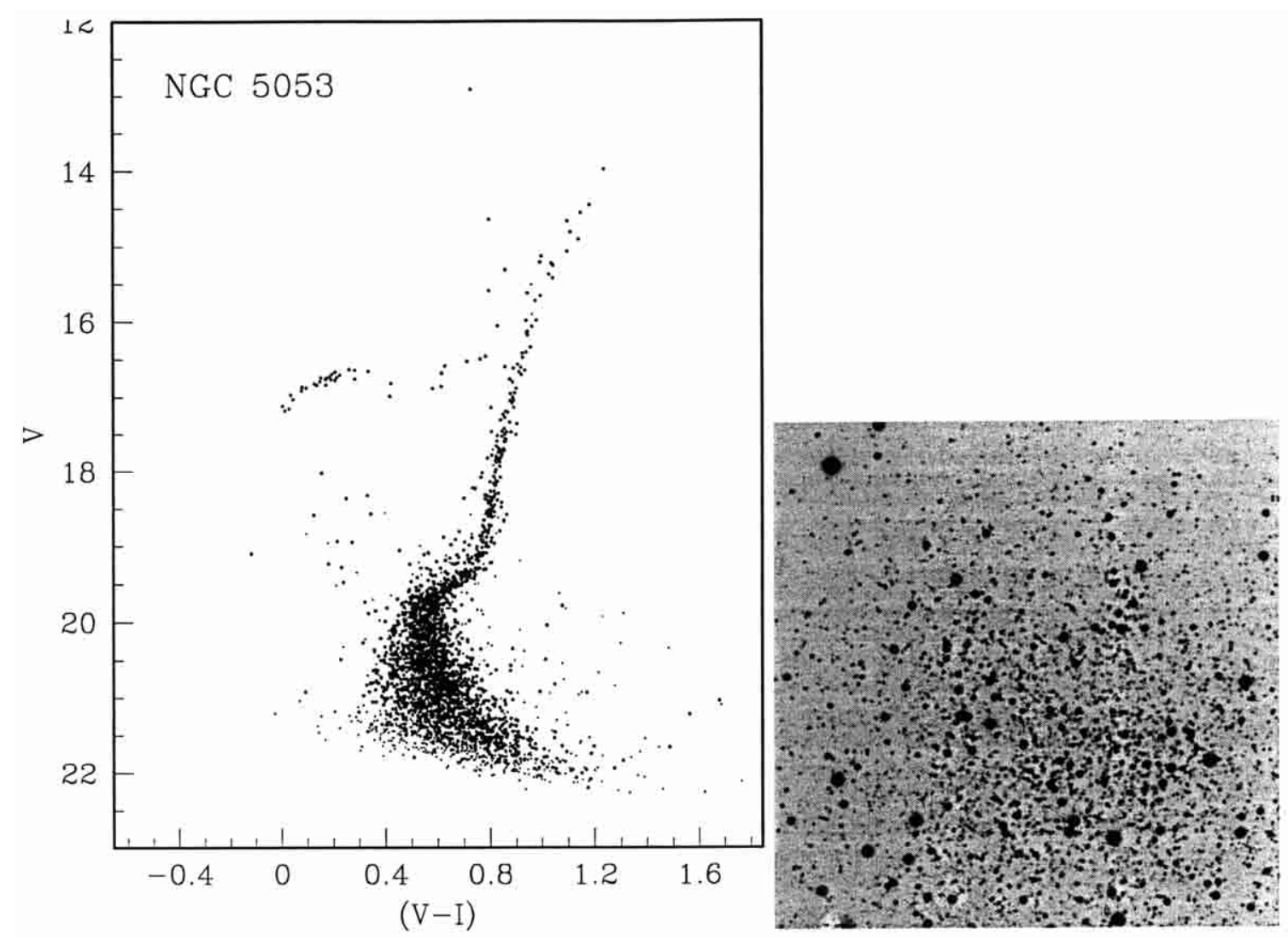

Fig. 4. CMD and covered field for NGC 5053

variable stars in the $B$ and $V$ bands. Their CMD has a limiting magnitude $V \sim 21$, is less populated (probably a factor of two, based on the number of BHB stars), since the MS stars are very sparse. An excellent CMD in the $V$ and $I$ filters is presented by Johnson \& Bolte (1998). Our field does not match that covered by them. However, the fiducial line representing their CMD well matches our diagram, with a small zero point difference (of the order of 0.02 magnitudes in $V$ ) in the RGB: a difference within the uncertainties of our absolute calibration. Recently, Davidge \& Courteau (1999) published $J H K$ data for four clusters, including M 3. Their CMD extends from the brightest stars down to the (sparse) MS. A large sample of M 3 stars $(\sim 37000)$ has been measured in the $V$ and $I$ bands by Rood et al. (1999), combining groundbased and HST data extending from the cluster center to the outer radius. This CMD is very clean as the high HST resolution limits crowding effects in the cluster center. Notice that the RR-Lyrae stars were identified and removed by the authors.

Our photometry covers the cluster from the tip of the RGB to the $23^{\text {rd }}$ magnitude ( $\sim 18750$ stars $)$, under quite good seeing conditions. The HB bimodality is clearly visible. Stars spread above the MS turn-off and blue-ward from the RGB are at small distances from the cluster center. If these stars were not plotted, the CMD would become very well defined (larger dots). Moreover, a large number of RR-Lyrae stars are present in our diagram, and our dispersion in color (see the MS, for example) is smaller than that present on the Rood et al. (1999) diagram.

NGC 5466. (Fig. 6)

NGC 5466 has one of the lowest central densities among the GGCs. Previous CMDs are not very recent; Nemec \& Harris (1987) present photographic and CCD data, with the CCD CMD extending from $V \sim 21$, to just below the HB (not present). We have not found more recent CMDs for this cluster.

We present a CMD from the RGB tip down to $V \sim$ 22.5 covering $\sim 5000$ stars. NGC 5466 resembles in many aspects NGC 5053, including the metal content, and also their CMDs look very similar.

\section{NGC 5904 (M 5). (Fig. 7)}

The globular cluster M 5 harbors one of the richest collection of RR-Lyrae stars in the Galaxy. It also hosts one of the only two known dwarf novae in GGCs.

The first CCD CMD for this cluster is published by Richer \& Fahlman (1987), who present deep $U, B$, and $V$ photometry. They give a well defined diagram, but poorly populated on the RGB and the HB. More recently, Sandquist et al. (1996) present $B, V$, and $I$ photometry for more than 20000 stars in M 5, and an excellent 


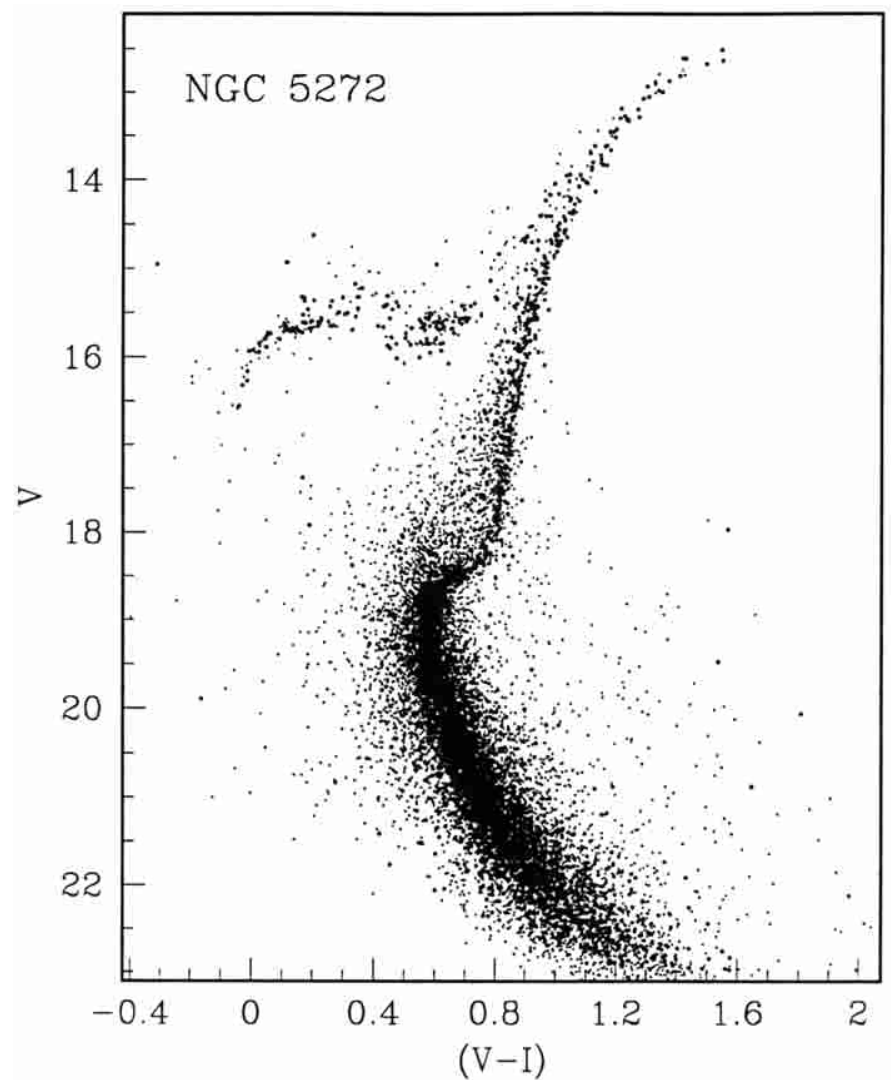

Fig. 5. CMD and covered field for NGC 5272 (M 3)
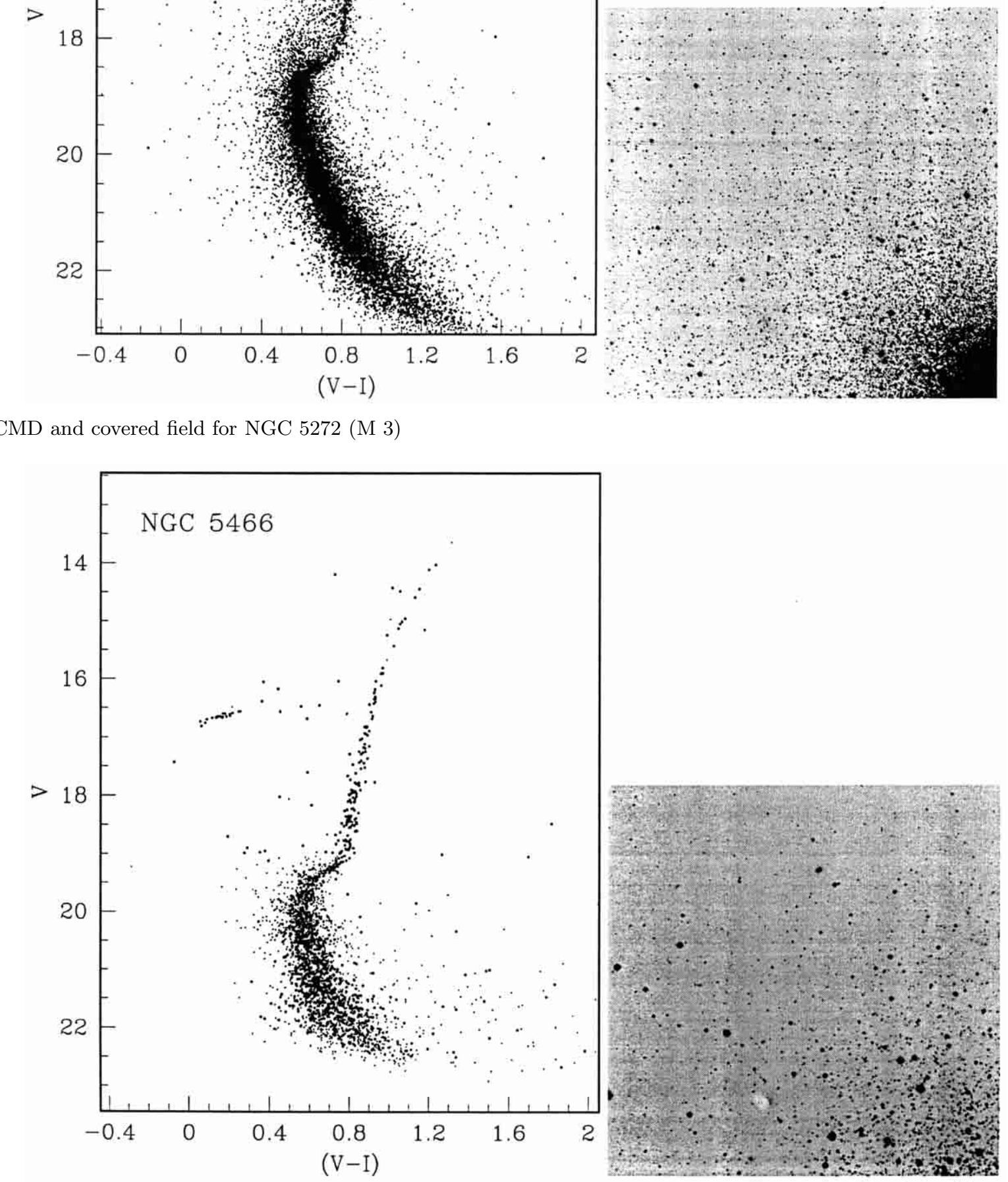

Fig. 6. CMD and covered field for NGC 5466 


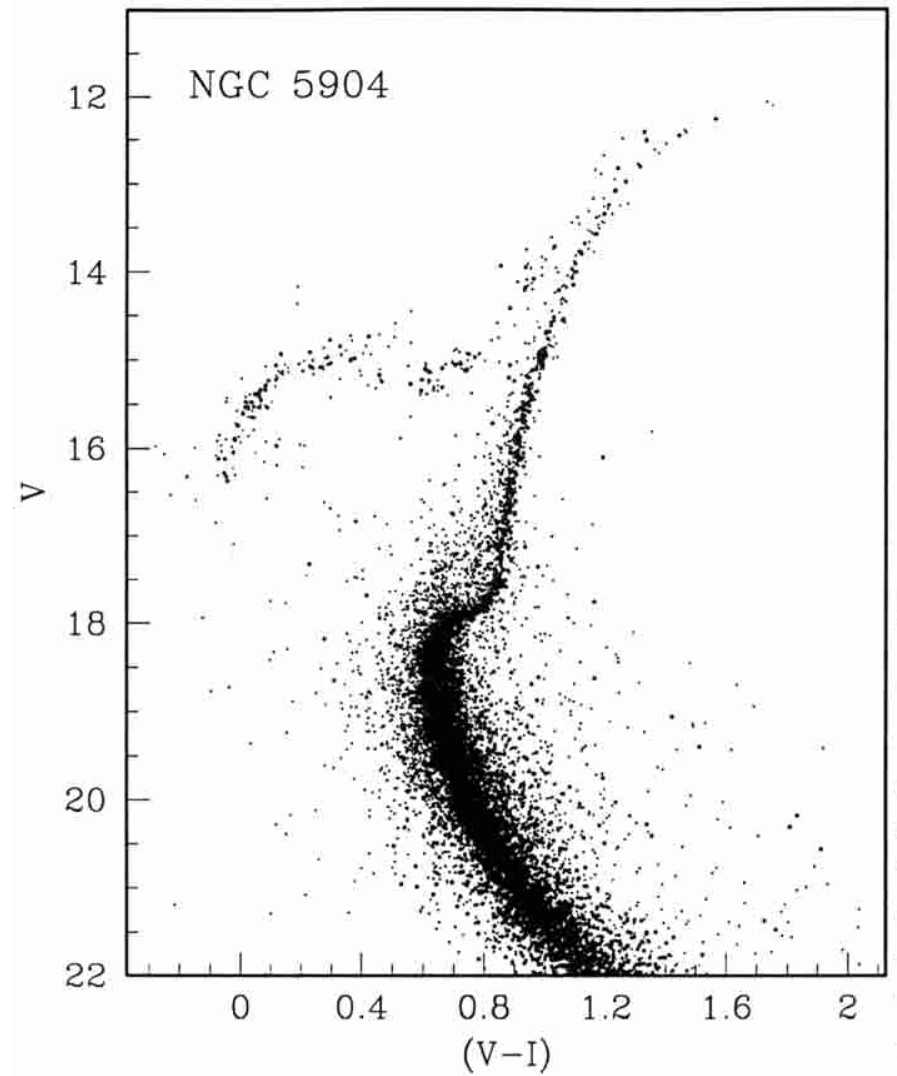

Fig. 7. CMD and covered field for NGC 5904 (M 5)
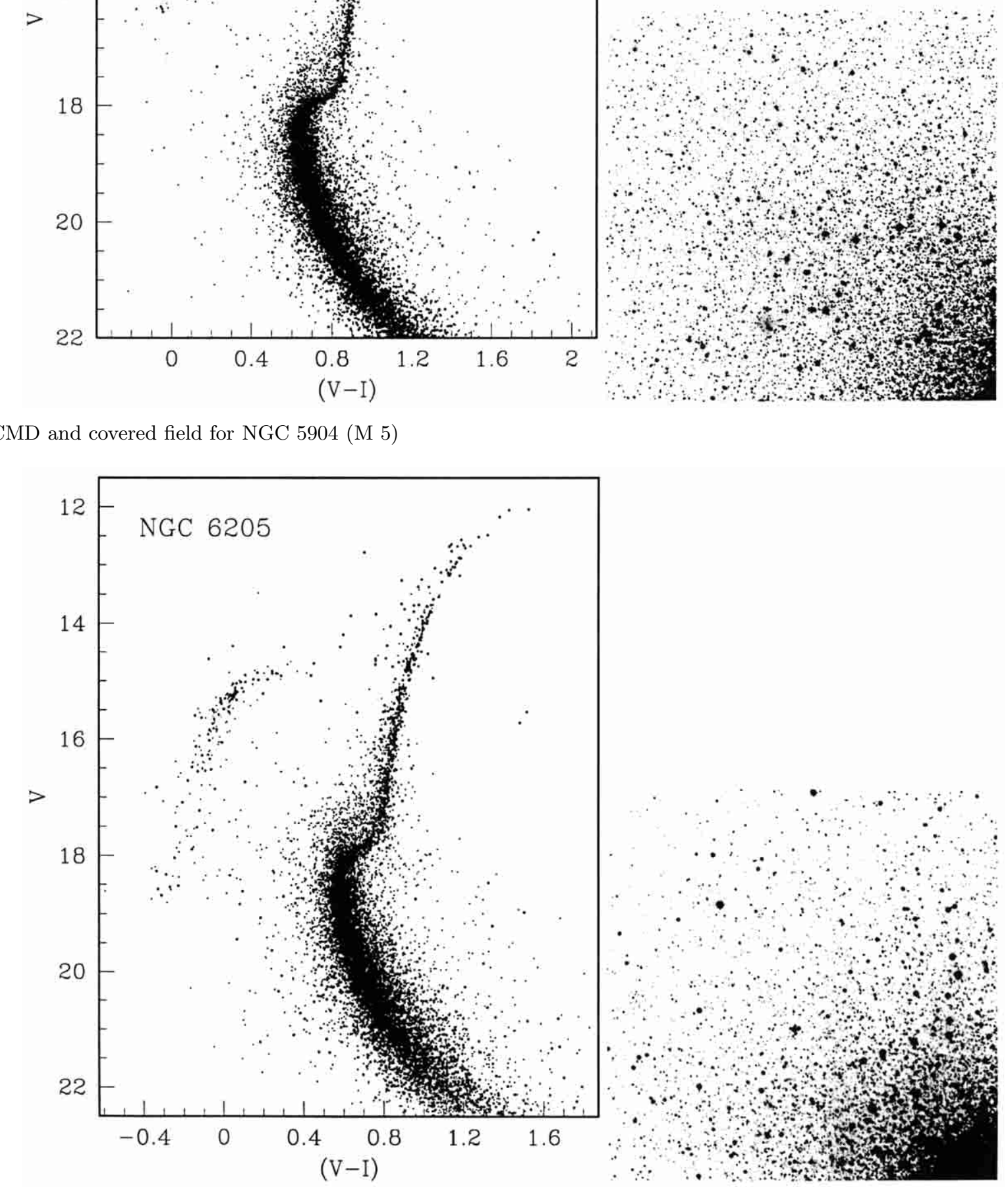

Fig. 8. CMD and covered field for NGC 6205 (M 13) 

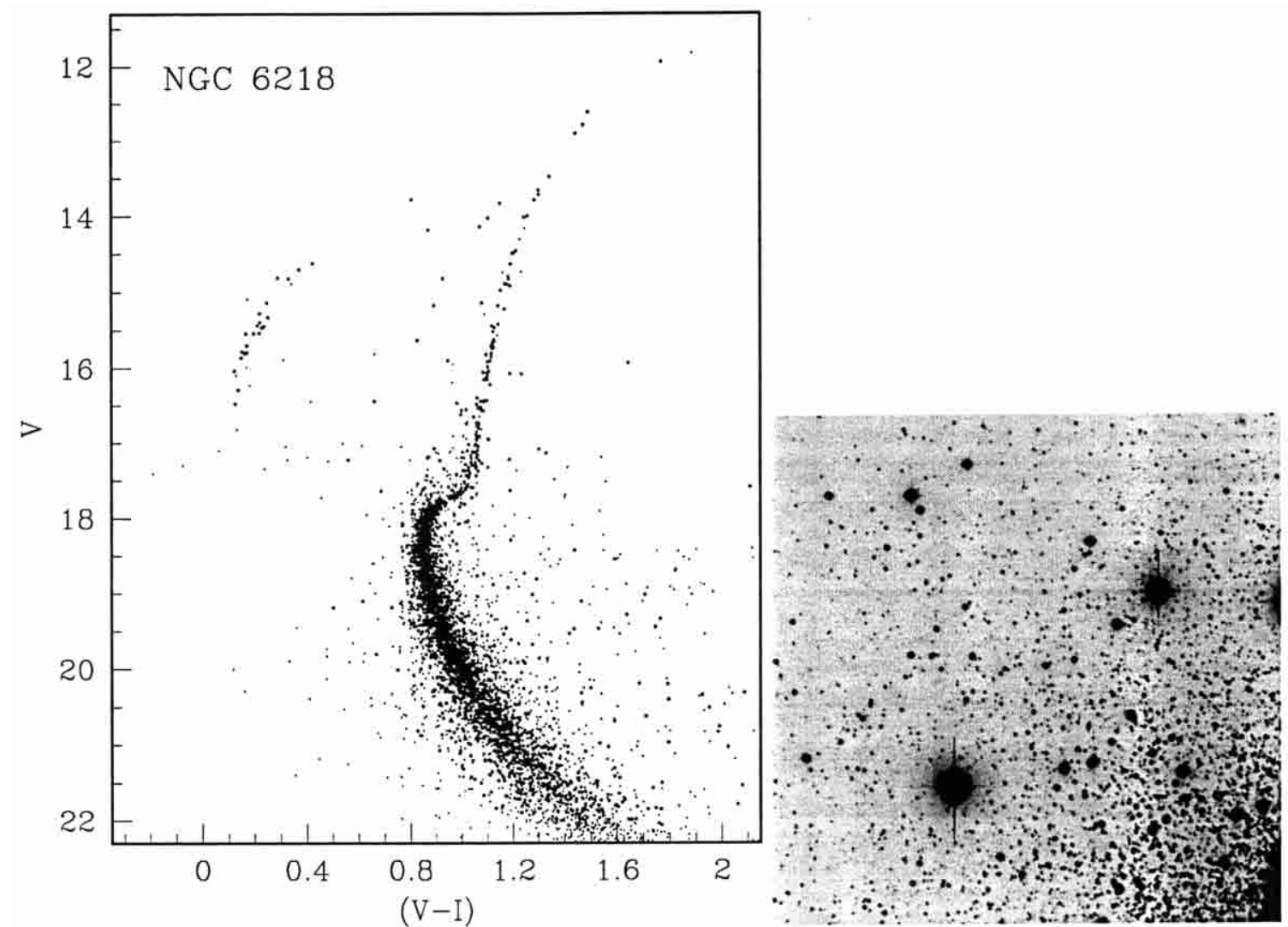

Fig. 9. CMD and covered field for NGC 6218 (M 12)

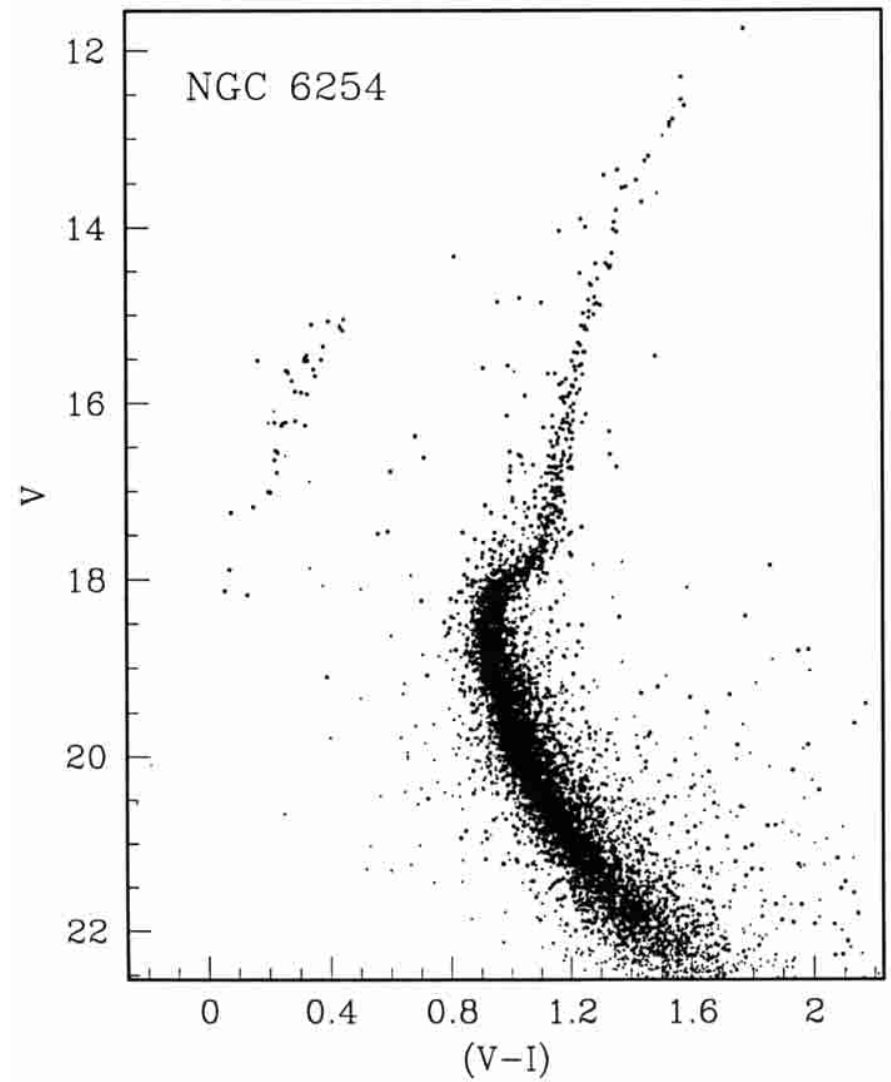

Fig. 10. CMD and covered field for NGC 6254 (M 10) 

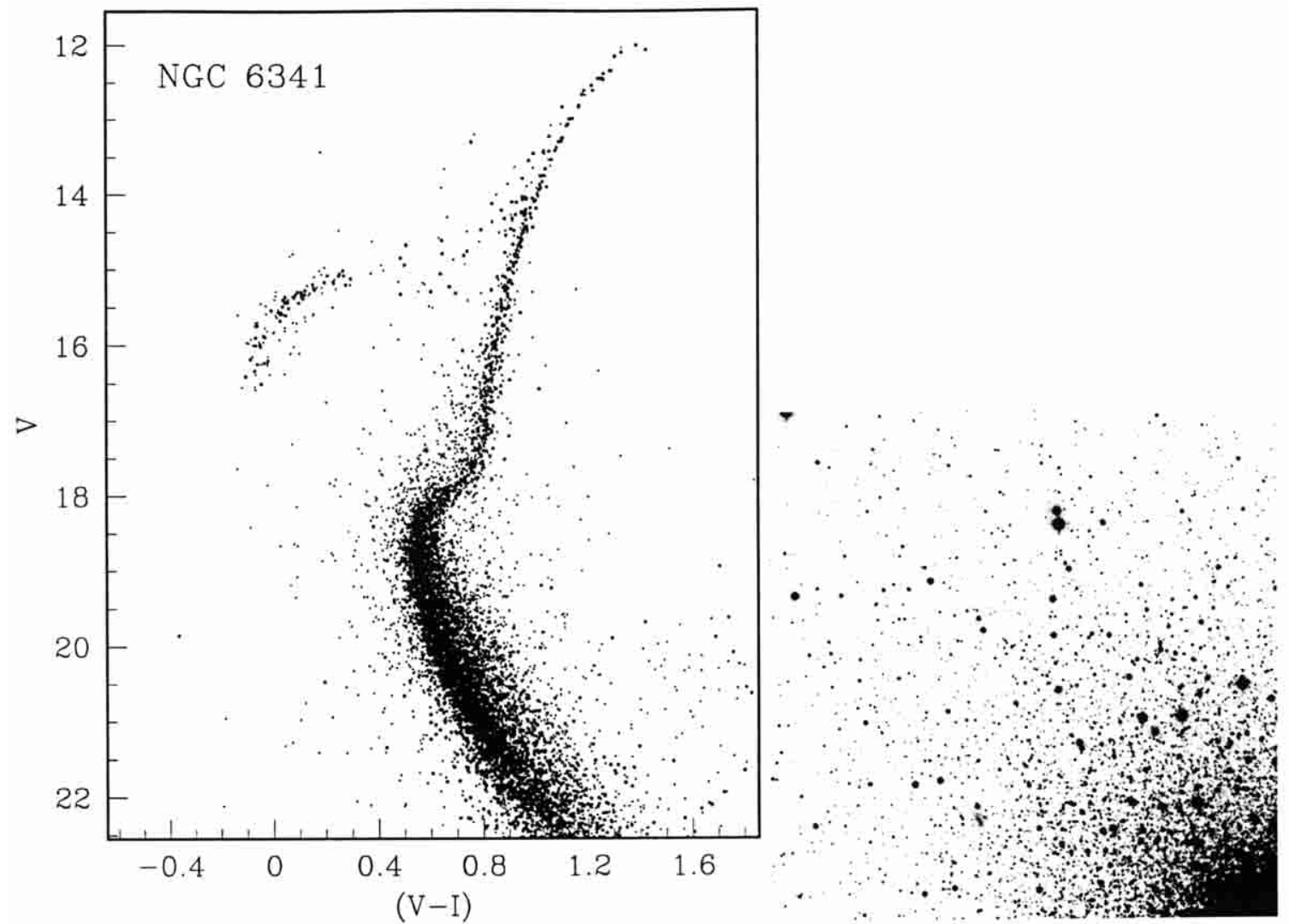

Fig. 11. CMD and covered field for NGC 6341 (M 92)
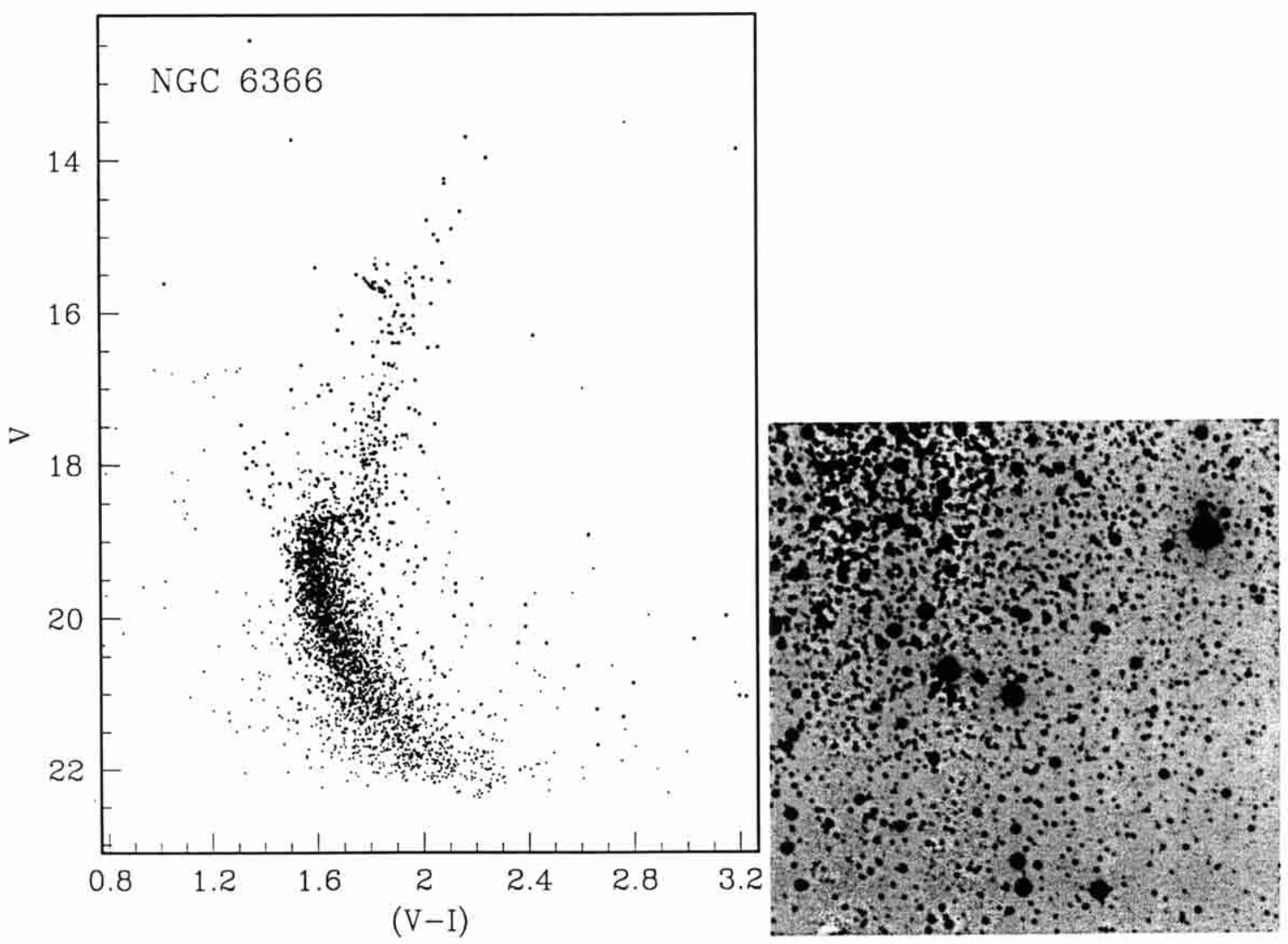

Fig. 12. CMD and covered field for NGC 6366 


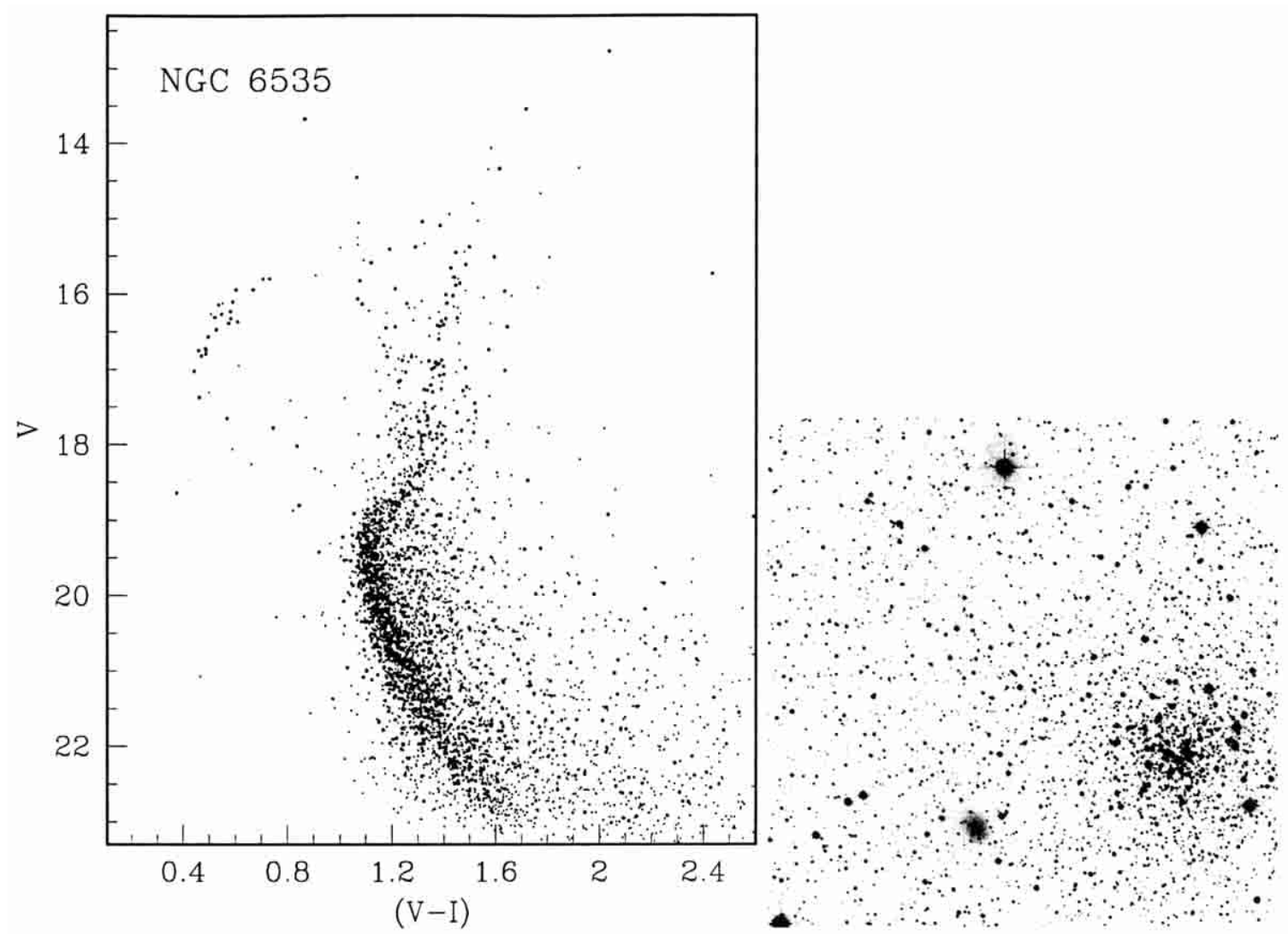

Fig. 13. CMD and covered field for NGC 6535

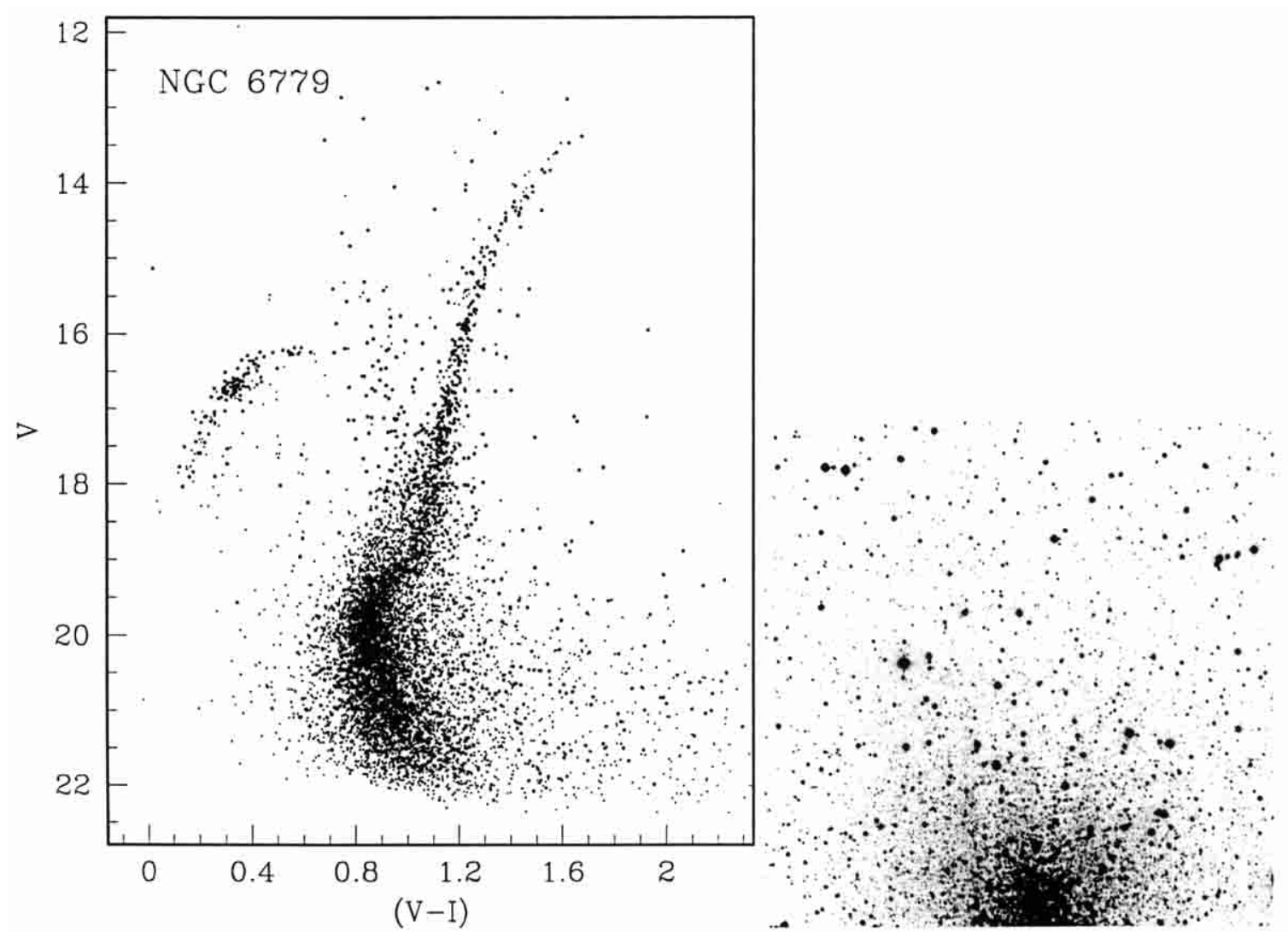

Fig. 14. CMD and covered field for NGC 6779 (M 56) 

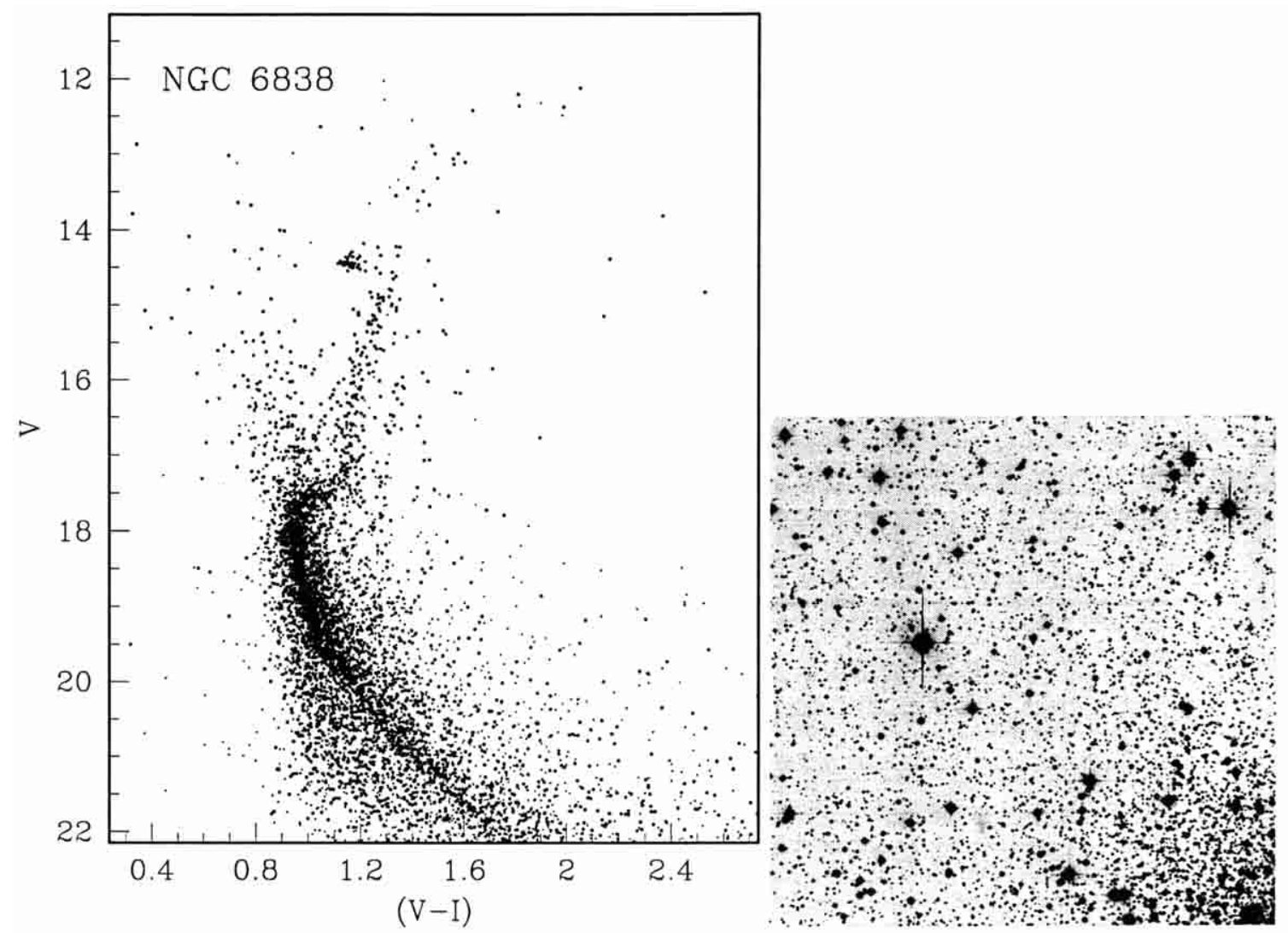

Fig. 15. CMD and covered field for NGC 6838 (M 71)
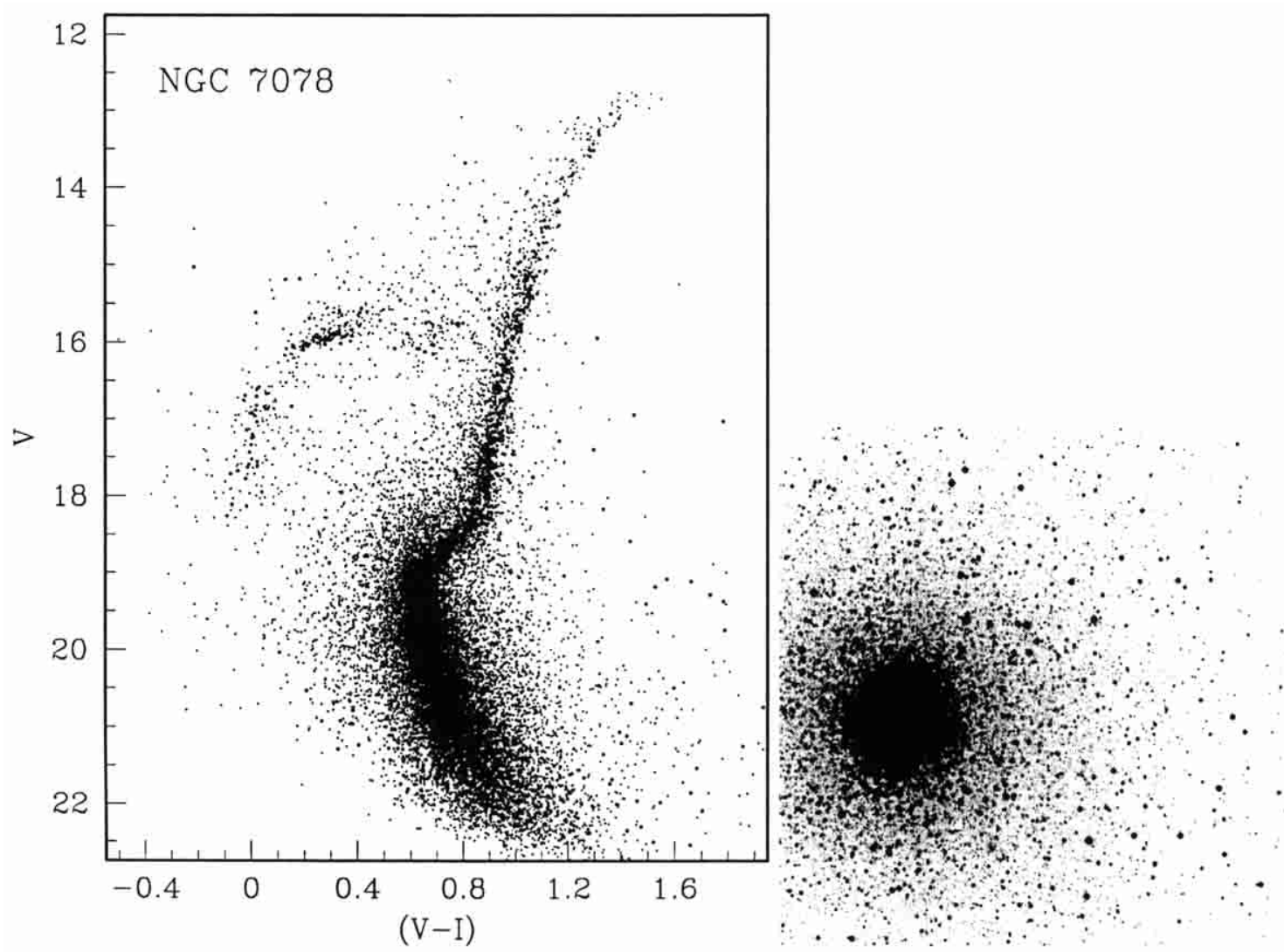

Fig. 16. CMD and covered field for NGC 7078 (M 15) 
CMD extended down to $V \sim 22$. The latest ground-based study is in Johnson \& Bolte (1998), who presented very good $V$ and $I$ photometry for this cluster. They compare their photometric calibration with that of Sandquist et al. (1996), with an uncomfortable trend with magnitude and an offset that increases with decreasing brightness. Recently, HST data have been published by Drissen \& Shara (1998), who studied the stellar population and the variable stars in the core of the cluster. Again, we compared our photometry with the fiducials from Johnson \& Bolte (1998), finding a good agreement within the errors.

Also NGC 5904 has been observed during quite good seeing conditions. The CMD extends by $\geq 4$ magnitudes below the TO, and includes $\sim 18300$ stars. All the CMD branches are well defined. In particular, note the perfect distinction between the $\mathrm{AGB}$ and the RGB, quite rare even in recent CMDs also for other clusters, and the extended blue straggler sequence.

\section{NGC 6205 (M 13). (Fig. 8)}

As already mentioned, M 13 and M 3 are a classical second parameter couple. M 13 has only a BHB, still, in several recent studies, it is found to be coeval with $\mathrm{M} 3$ (e.g. Paper III). In our CMD it shows a sparse EBHB that arrives to the MS TO magnitude.

Previous studies include Richer \& Fahlman (1986) who obtained $U, B$, and $V$ CCD photometry for this cluster from $\sim 2$ magnitudes above the TO to $V \sim 23$. Their CMD is very well defined, but for a very small sample of stars. Moreover, just 6 RGB stars under the HB are present. More recently, CMDs are presented again by Johnson \& Bolte (1998), with their ( $V, I)$ CMD and our one perfectly overlapping within the errors. Paltrinieri et al. (1998) present $B, V$ photometry for $\sim 5500$ stars from the RGB tip to about 2 magnitudes below the MS TO. Their photometry is more sparse in the SGB and specially in the MS. Davidge \& Courteau (1999) have published a JHK photometry. They cover basically the more evolved branches of the diagram.

\section{NGC 6218 (M 12). (Fig. 9)}

The only two CCD studies that we have found in the literature for this cluster are those of Sato et al. (1989), who presented $U B V$ data for the MS and SGB region in a poorly populated CMD, and Brocato et al. (1996), who present a sparse $B$ and $V$ CMD from the tip of the RGB to a few magnitudes below the cluster TO.

Our CMD is not very populated $(\sim 7100$ stars measured), but the main lines of the RGB, BHB, SGB and MS are very well defined. It is noticeable that, despite its intermediate metallicity, this cluster shows only a BHB, resembling in some way M 13.
NGC 6254 (M 10). (Fig. 10)

Hurley et al. (1989) present the first CCD CMD of this cluster in the $B$ and $V$ bands, covering from the RGB (poorly populated) to $V \sim 21.5$. However, already in this diagram the remarkable EBHB is clearly visible. Recently, Piotto \& Zoccali (1999) present HST data for the MS of this clusters and analyze the cluster luminosity function, using the present data for the evolved part of the CMD.

Our CMD is well defined and extends for more than 4 magnitudes below the TO, covering a total of $\sim 13000$ stars. The cluster has only a BHB, and we confirm that it is extended. In many respects, the CMD of M 10 resembles that of M 13.

\section{NGC 6341 (M 92). (Fig. 11)}

M 92 is one of the most metal-poor and one of the best studied globular clusters in the Galaxy. It was (together with M 3) the first GGC to be studied down to the TO (Arp et al. 1952, 1953). Since then, many CMDs have been built for NGC 6341. The first CCD photometry is presented by Heasley \& Christian (1986). They obtain a CMD down to $V=22$ in the $B$ and $V$ filters, but poorly populated. Another exhaustive work on M 92 is presented by Stetson \& Harris, who present a deep $B$ and $V$ CMD with a very well defined MS, but still poorly populated in the evolved part of the diagram. More recently, Johnson \& Bolte (1998) present an excellent $V$ and $I$ diagram of M 92 where the principal sequences are very well defined, but there is still a small number of stars in the evolved regions. $J H K$ photometry of this cluster is presented by Davidge \& Courteau (1999). Piotto et al. (1997) present a deep CMD from HST/WFPC2 extended down to $\sim 0.15 M_{\odot}$.

Our diagram, with $\sim 13900$ stars measured, is well defined and extends from the RGB tip to about 4 magnitudes below the MS TO.

\section{NGC 6366. (Fig. 12)}

This cluster is somehow peculiar. While located in the disk, and being a metal-rich cluster (as M 71 or 47 Tucanae), it has a kinematics typical of a halo cluster. It is also highly reddened, and its CMD is affected by some differential reddening.

Alonso et al. (1997) present the only other CCD $B$ and $V$ CMD existent for this cluster. NGC 6366, together with NGC 5053, are the only two clusters that were observed under not exceptionally good seeing conditions. Still, all the sequences in the CMD can be identified (apart from the upper RGB), including what seems to be a well populated blue straggler sequence. The $\mathrm{HB}$ is very red, as expected on the basis of the metallicity, and tilted. We measured a total of $\sim 5500$ stars for this cluster.

NGC 6535. (Fig. 13)

To our knowledge, Sarajedini (1994) has published the 
only previous CCD study of this cluster: a $B$ and $V$ CMD down to $V \sim 21$. His stellar population is slightly smaller than ours for this range of magnitudes (we reach $V \sim 23$ ). NGC 6535 is the least luminous object of our northern sample, and probably the one with the smallest number of stars. We measured $\sim 7800$ stars for this cluster. Its RGB is identifiable, but not clearly defined, due also to field star contamination. Its CMD somehow resembles the CMD of NGC 6717 (Paper I).

NGC 6779 (M 56). (Fig. 14)

We have not found any previous CCD study on this cluster. Our CMD is well defined, though it is slightly contaminated by foreground/background stars. The broadening of the SGB-RGB might suggest the existence of some differential reddening. The distribution of the stars along the BHB seems to be not homogeneous, with the possible presence of a gap. The total of measured stars was of $\sim 11300$.

\section{NGC 6838 (M 71). (Fig. 15)}

As suggested also by its CMD, M 71 is a metal rich cluster, similar to 47 Tuc (Paper I). Our CMD is well defined and extends for more than 4 magnitudes below the TO, covering a total of $\sim 12500$ stars. The cluster has only a RHB, and the upper part of the RGB is not very well defined. This cluster is located close to the Galactic plane, and this explains the contamination by disk stars clearly visible in the CMD. It is very bright and relatively nearby.

Despite this, there is no CMD in the literature after Hodder et al. (1992). They present a good $B$ and $V$ diagram, less populated than ours, reaching $V=22$.

Previous CCD studies are in Richer \& Fahlman (1988), who present $U, B, V$ photometry for the main sequence, down to $V=22(U=25)$. No evolved stars are present in this work.

NGC 7078 (M 15). (Fig. 16)

This cluster has been extensively studied in the past, both with groundbased facilities and a large number of HST observations.

HST studies include Stetson (1994) and Yanny et al. (1994), were a CMD of the cluster center is presented. The CMD does not arrive to the MS TO, and is quite disperse. Conversely, Sosin \& King (1997) and Piotto et al. (1997) present and extraordinarily well defined MS, but no evolved stars are present.

The most recent ground-based study is the composite CMD of Durrell \& Harris (1993) based on CCD data from two telescopes. This is the kind of problem that we try to avoid with the present catalog.

Our diagram is well populated $(\sim 27000$ stars $)$ from the RGB tip down to $V=22.5$. The CMD features are better identifiable when a radial selection, avoiding the clusters center, is done. The CMD in Fig. 16 gives the visual impression that there are three distinct groups of stars in the HB. The third possible group, on the red side of the RR Lyrae gap is surely a statistical fluctuation in the distribution of the RR Lyrae magnitudes and colors at random phase. It is present neither in the CMDs of M 15 in the above quoted works nor in our CMD of a larger stellar sample, with more accurate photometry from our HST data base.

Acknowledgements. This paper has been partially supported by the Ministero della Ricerca Scientifica e Tecnologica under the program "Treatment of large format astronomical images" and by CNAA.

\section{References}

Alonso A., Salaris M., Martinez Roger C., Straniero O., Arribas S., 1997, A\&A 323, 374

Arp H.C., Baum W.A., Sandage A.R., 1952, AJ 57, 4

Arp H.C., Baum W.A., Sandage A.R., 1953, AJ 58, 4

Brocato E., Buonanno R., Malakhova Y., Piersimoni A.M., 1996, A\&A 311, 778

Carretta E., Gratton R., 1997, A\&AS 121, 95

Davidge T.J., Courteau S., 1999, AJ 117, 1297

Drissen L., Shara M.M., 1998, AJ 115, 725

Durrell P.R., Harris W.E., 1993, AJ 105, 1420

Fahlman G.G., Richer H.B., Nemec J., 1991, ApJ 380, 124

Harris W.E., 1996, AJ 112, 1487

Heasley J.N., Christian C.A., 1986, ApJ 307, 738

Heasley J.N., Christian C.A., 1991a, AJ 101, 967

Heasley J.N., Christian C.A., 1991b, ASP Conf. Ser. 13, 266

Hodder P.J.C., Nemec J.M., Richer H.B., Fahlman G.G., 1992, AJ 103, 460

Hurley D.J.C., Richer H.B., Fahlman G.G., 1989, AJ 98, 2124

Johnson J.A., Bolte M., 1998, AJ 115, 693

Kaluzny J., Hilditch R.W., Clement C., Rucinski S.M., 1998, MNRAS 296, 347

Laget M., Ferraro F.R., Paltrinieri B., Fusi Pecci F., 1998, A\&A 332, 93

Landolt A.U., 1992, AJ 104, 340

Lee Y.W., 1990, ApJ 363, 159

Nemec J.M., Harris H.C., 1987, ApJ 316, 172

Nemec J.M., Cohen J.G., 1989, ApJ 336, 780

Paltrinieri B., Ferraro F.R., Carretta E., Fusi Pecci F., 1998, MNRAS 293, 434

Piotto G., Cool A., King I., 1997, AJ 113, 1345

Piotto G., Zoccali M., 1999, A\&A 345485

Richer H.B., Fahlman G.G., 1986, ApJ 304, 273

Richer H.B., Fahlman G.G., 1987, ApJ 316, 189

Richer H.B., Fahlman G.G., 1988, ApJ 325, 218

Rood R.T., Carretta E., Paltrinieri B., et al., 1999, ApJ 523, 752

Rosenberg A., Saviane I., Piotto G., et al., 1998, AJ 115, 648

Rosenberg A., Saviane I., Piotto G., Aparicio A., 1999, AJ 118, 2306 (Paper III)

Rosenberg A., Piotto G., Saviane I., Aparicio A., 2000, A\&AS 144, 5 (Paper I)

Rutledge G.A., Hesser J.E., Stetson P.B., 1997, PASP 109, 907 
Sandquist E.L., Bolte M., Stetson P.B., Hesser J.E., 1996, ApJ 470,910

Sarajedini A., 1994, PASP 106, 404

Sarajedini A., Milone A.A., 1995, AJ 109, 269

Sato T., Richer H.B., Fahlman G.G., 1989, AJ 98, 1335

Saviane I., Rosenberg A., Piotto G., Aparicio A., 2000, A\&A 355, 966 (Paper IV)
Sosin C., King I., 1997, AJ 113, 1328

Stetson P.B., Harris W.E., 1988, AJ 96, 909

Stetson P.B., 1994, PASP 106, 250

Thuan T.X., Gunn J.E., 1976, PASP 88, 543

Yanny B., Guhathakurta P., Bahcall J.N., Schneider D.P., 1994, AJ 107, 1745

Zinn R., West M.J., 1984, ApJS 55, 45 